quirement. In the end, a compromise was reached: before the Conference, Administrations were permitted to waive the requirement for operation above $144 \mathrm{MHz}$, and this limit was changed to $30 \mathrm{MHz}$. Only one amateur band, at $50 \mathrm{MHz}$, is affected by this change.

The ITU Radio Regulations set out some rather complex and detailed procedures to be followed by Administrations which intend to authorize the operation of space services. The Amateur-Satellite service is not exempt from those procedures; however, the nature of the service makes it impossible to comply with the letter of the regulations. Unlike stations in other space services, amateur stations throughout the world have free access to amateur satellites and the sponsors of the satellites have no way (and no reason) to determine the identity of each and every user. Thus it is impossible to provide details on the location, equipment, antennas, etc., of each and every Earth station. Resolution 642 (BV) eases the burden of notification for the Amateur-Satellite service, making optional the notification of amateur Earth station characteristics.

Another resolution of particular interest to amateurs is Resolution 640 (BN), concerning the international use of radiocommunications in the event of natural disasters. This resolution notes the value of the Amateur service in natural disasters and establishes guidelines for the use of the amateur bands by nonamateur stations responding to such disasters.

\section{CONCLUSION}

WARC-79 was an event of immense importance to the one million radio amateurs worldwide. In general, and particularly in the areas of high-frequency and amateur-satellite allocations, amateur radio is in an improved position as a result of the Conference. These improvements did not occur by happenstance; they were the result of years of coordinated preparations, mostly by an international group of dedicated volunteers working to influence the positions of their national Administrations. These preparations, and the long tradition of the Amateur service as a valuable technical and publicservice communications resource, earned for it the support of enough national Administrations to assure a favorable outcome. Amateur radio's preparations for WARC-79 can serve as a model for future ITU Administrative Radio Conferences.

\section{REFERENCES}

[1] ITU, Final Acts of the World Administrative Radio Conference Geneva, 1979. Geneva, Switzerland, 1979, para. N3044/78.

[2] E. W. Terrie, "Amateur radio in the United States and Canada, 1980," Florida State University Institute for Social Research, Tallahassee, FL, Sept. 1980.

\title{
Future ITU Radio Conferences
}

\author{
FRANCIS S. URBANY
}

\begin{abstract}
The 1979 World Administrative Conference (WARC-79) was tasked, by the terms of its agenda, to consider the need for holding future radio conferences to deal with specific radio services. As a consequence, WARC-79 identified an extensive program of 11 world or regional conferences to be convened in the years immediately ahead. The most challenging and complex conferences will be two WARC's (in two sessions each) to consider the planning of the HF Broadcasting service and the planning of space services utilizing the geostationary-satellite orbit. Both conferences will deal with means of assuring equitable access to the radio spectrum (and to the orbit in the case of space services) by all administrations.
\end{abstract}

Manuscript received May 1, 1981; revised May 11, 1981

The author is with the United States Department of Commerce, National Telecommunications and Information Administration, Washington, DC 20005.

\section{INTRODUCTION}

$\mathbf{A}$ MONG THE more imposing legacies handed down by the 1979 International Telecommunication Union (ITU) World Administrative Radio Conference (WARC-79) is a broad array of future conferences. In the decade of the 1980's the holding of some 11 conferences has been mandated as a result of decisions taken at the 1979 Conference. Even to observers familiar with past ITU radio conferences this seems, and is, a dramatic concentration of conference activity. Not that the period since 1959, when the last comparable world radio conference was held, was by any means barren of conference activity. In fact, during the intervening 20-year span some 8 world and several regional administrative radio conferences were con- 
ducted. But what is particularly striking about the future work flowing from WARC-79 is the fact that such an extensive panoply of future radio conferences was identified at one time.

Customarily, individual radio conferences have been scheduled as the need for making improved arrangements for dealing with the various radio communication services became evident. In discussions leading up to WARC.79 it became apparent that however broad and encompassing the goals of the conference were, the agenda of the 1979 Conference would nevertheless be limited in scope; and additional specialized radio conferences would be required to address individual service needs. For example, by terms of its agenda (except for allocations), WARC-79 was not permitted to revise the Radio Regulations dealing with single services such as the Mobile and Broadcasting services, etc., [1].

It was also thought that it would be helpful to the overall future work of the ITU if WARC-79 were to identify the whole range of radio conference activity required for the foreseeable future. In this way, budgetary impacts could be gauged, the work of the Union organized in an orderly fashion, and sufficient time allowed for the involved ITU organizations such as the International Frequency Registration Board (IFRB) and the International Radio Consultative Committee (CCIR), and for Administrations themselves to make adequate preparations.

Accordingly, a specific item was included in the agenda of WARC-79 calling upon the conference:

"to propose to the Administrative Council and to the next Plenipotentiary Conference a programme for convening future administrative Radio Conferences to deal with specific services." [1, para. 2.10].

Before examining the schedule of future conferences mandated by WARC-79 it would be useful to place in context the role of administrative radio conferences in the affairs of the Union. As provided for in the ITU Convention, administrative conferences constitute one of the principal means by which the Union conducts its work [2, Art. 7].

Administrative conferences are normally convened to consider specific telecommunications matters and are of two kinds:

1) World Administrative Conferences

2) Regional Administrative Conferences.

World administrative conferences undertake Revision of the Administrative Regulations (Telegraph Regulations, Telephone Regulations, Radio Regulations, and Additional Radio Regulations) or other matters of a worldwide character within the competence of the conference. In contrast, regional administrative conferences may consider only telecommunication questions of a regional nature. The decisions of world and regional administrative conference must be in conformity with the provisions of the ITU Convention and additionally, the decisions of regional administrative conferences must be in conformity with the provisions of the Administrative Regulations [2, Art. 7].

Given the dynamic nature of developments in radio communications, the administrative conferences constitute the principal mechanism by which the Radio Regulations are updated in response to evolving needs and changing conditions. Historically, administrative conferences have proven to be a practical, flexible means by which Administrations are able to adapt the international rules governing the use of the radio spectrum to meet changing requirements. As noted above, such conferences have been resorted to extensively over the past twenty years. Decisions taken at conferences need not be, and in fact are not, fixed for all time but can be modified and updated as changing conditions dictate. Importantly then, the administrative conference enables the ITU to be a living, dynamic institution that is responsive to the needs of its members.

Against this background, let us examine the actions taken at WARC-79 with respect to laying out a program of future radio conferences as called for in the 1979 Conference agenda. The need for the following world and regional conferences was identified [3]:

\section{World Administrative Conferences}

World Administrative Radio Conferences for the Mobile Services (Resolution 202);

World Administrative Radio Conference for the Planning of the HF Bands Allocated to the Broadcasting Service (2 sessions: 1983 and 1984, Resolution 508);

World Administrative Radio Conference on the Use of the Geostationary-Satellite Orbit and the Planning of Space Services utilizing it ( 2 sessions: 1984 and 1985, Resolution 3).

\section{Regional Administrative Conferences}

Final Session, Region 2, Medium Frequency Broadcasting Conference (November 1981-arranged for prior to WARC79);

Region 2 Broadcasting-Satellite Planning Conference (1983, Resolution 701);

Planning Conference for Sound Broadcasting in the 87.5$108-\mathrm{MHz}$ band for Region 1 and Certain Countries Concerned in Region 3 (2 sessions: not later than 1983, Resolution 510);

Conference to Draw up Agreements and Associated Plans for Feeder Links to Broadcasting-Satellites Operating in the 12-GHz Band in Regions 1 and 3 (Resolution 101);

Conference to Establish criteria for the Shared Use of the VHF and UHF Bands Allocated to Fixed, Broadcasting, and Mobile Services in Region 3 (Resolution 702);

Conference to Revise the Plan of the Copenhagen Convention 1948, for the European Maritime Area-Region 1 (Recommendation 602);

Conference to Review and Revise the Provisions of the Final Acts of the African VHF/UHF Broadcasting Conference, Geneva, 1963 (Resolution 509);

Planning Conference for Broadcasting in the Band $1605-$ $1705 \mathrm{kHz}$ in Region 2 (not later than 1985; Recommendation 504). 
Several observations about the schedule of these conferences are in order. In a number of cases, a suggested date for holding a particular conference has been indicated and is shown in parathensis (together with the number of the relevant resolution or recommendation which contains the suggested conference agenda). For the most part, the conferences are "planning" conferences which look toward the development in some form or other of more detailed ground rules for specifying use of the services and frequency bands involved. In several instances, two-session conferences are called for in which the first session is to determine the technical basis for the specific planning measures which are to be adopted at the second session. And finally, the majority of the conferences deal with some aspect of the broadcasting service, both terrestrial and space, in various portions of the radio spectrum. A detailed examination of each of the conferences is not practical here; instead, this paper will highlight those conferences of broadest involvement and significance.

\section{WORLD ADMINISTRATIVE RADIO CONFERENCES}

\section{Mobile-Services WARC}

Prior to WARC-79, the need to revise those parts of the Radio Regulations dealing with the Mobile services was discussed by the ITU Administrative Council, but the matter was referred to WARC-79 for consideration [4] Provisions relating to the Mobile service constitute about half the volume of the Radio Regulations and cover the Aeronautical, Maritime, and Land-Mobile services-including terrestrial and space aspects.

In part, the desire to revise the Mobile service regulations stems from the need to complete an earlier undertaking to rearrange the presentation of certain portions of the Radio Regulations so as to make them easier to use [1, para. 2.7]. The rearrangement effort organized the Mobile services into separate chapters in the regulations and resulted in the duplication of many provisions. Moreover, the rearrangement effort did not extend to making substantive changes to the regulations.

Additionally, WARC-79 made frequency-allocation changes and there was general recognition of the need to harmonize various portions relating to the individual Mobile services. These developments, coupled with the need to provide for improved distress and safety arrangements, thus gave rise to the call for a world conference to undertake a comprehensive revision of the Radio Regulations relating to the Mobile services. The Administrative Council decided at its 1980 session to split the Mobile conference into two separate conferences. The first conference will be held in 1982, and it will concentrate primarily on the safety needs which can be satisfied by the Mobile services. A full treatment of the general needs of the Mobile services will be given at the second conference which will be held in 1986 or thereafter.

\section{HF Broadcasting and Space Services WARC}

In contrast to the 1982 Mobile services WARC which now has a relatively limited mandate and, in practice, will likely be of primary interest to maritime nations, the future WARC dealing with the planning of $\mathrm{HF}$ (short-wave) broadcasting and space services undoubtedly will attract universal interest and participation by the Union's 155 member Administrations. Although HF Broadcasting has been long established (by comparison with space communication which is relatively recent), technological innovations have benefitted both services. Consequently, both conferences will address a fundamental theme: namely, how to assure access to the spectrum, and (additionally, in the case of space communications, access to the geostationary-satellite orbit) in a manner that equitably accommodates the needs of all nations. Given the importance attached to short-wave broadcasting and space communications, these two conferences unquestionably will be the most challenging (and indeed difficult) of all those listed on the ITU's future work program. This difficulty and complexity stems from not only the technical aspects which must be considered but also from the social, economic, and political dimensions inherent in achieving acceptable solutions.

With respect to HF broadcasting, WARC-79 basically dealt only with frequency-allocation matters. Nontheless, this was a highly controversial issue in and of itself, and it set the stage for future consideration of the whole range of factors bearing on HF broadcasting. Due to the congestion in the broadcasting bands as a result of increasing use over the past 20 years, many proposals were made at WARC to expand HF broadcasting allocations. Among those advocating expansion in addition to the United States, were a number of other developed countries which also have significant international short-wave broadcasting activities. Owing to the extensive use made by developing countries of the HF Fixed services for domestic purposes, there was general reluctance on their part to release Fixed service spectrum to permit expansion for Broadcasting. Also at issue was the concern by the developing nations that they would be assured future access to the expanded bands (as well as to existing bands). Ultimately, an accommodation was reached. Allocations for HF Broadcasting were increased by approximately one third, and a two-session WARC was to be convened for the purpose of planning the use of the HF bands allocated to the Broadcasting service.

The increase in allocations was viewed as inadequate by a number of countries, both developed and developing (including the United States) which entered protocol statements to the WARC-79 Final Acts that the additional spectrum was insufficient to enable the broadcasting conference to successfully plan all frequency bands, particularly those at 6 and $7 \mathrm{MHz}$. Accordingly, the countries reserved their right to take the necessary steps to meet their HF broadcasting needs in the absence of an adequate plan [5].

The use of the existing HF broadcasting allocations is governed by the provisions of Article 17 of the Radio Regulations [6]. These provisions provide for "seasonal" schedules to be made up four times a year to account for varying radio propagation conditions, and they amount to a flexible shortterm planning procedure. Although some frequency assignment features of the Radio Regulations are characterized as "first-come-first-served," no priority is accorded under Article 17 to any Administration by reason of any previous use of a particular frequency. Nevertheless, developing countries view the existing procedures with concern; and they consider that the procedures, in practice, work to their detriment. Article 17 was not on the WARC-79 agenda and, therefore, there was no 
systematic, in-depth review of its operation or an examination of the possibilities for its improvement. Consequently, it will be a subject for consideration at the broadcasting conference.

The existing Article 17 provisions were developed in 1959 after numerous previous attempts to draw up an HF broadcasting plan failed-due primarily to the fact that requirements submitted by the Administrations could not be accommodated within the existing allocations. If the HF Broadcasting WARC is to draw up a detailed plan, some solution to this ageless problem will have to be found. Quite apart from whatever planning formula the Broadcasting WARC adopts relating to access to broadcasting frequencies, the conference will address specific technical and operational aspects that could lead to improvements in spectrum use.

The first session of the conference is to establish the technical parameters to be used for planning, including appropriate limitations on power and the maximum number of frequencies to be used for the broadcasting of the same program to the same reception area. Importantly, while planning is to be based on double-sideband emissions (DSB) consideration is also to be given to the manner in which single-sideband (SSB) systems could be introduced progressively without impairing the DSB emissions. Hence, the first session of the conference is to draw up the specifications for an SSB system suitable for future HF broadcasting use. Adoption of SSB systems would have the effect of increasing the number of assignable channels within the allocations available for broadcasting. The conversion to SSB broadcasting is not a trivial problem, however, and necessarily it would need to be accomplished over a significantly lengthy period of time (10-15 years) to permit the phase-out of existing radio receivers and the development of low-cost SSB replacement receivers. The first session is also to decide the planning principles and methods to be adopted by the second session.

As with HF broadcasting, concern by ti.e developing countries over the adequacy of existing ground les for access to frequency allocations-in this case relating to space communications-gives rise to demands to conve e a future conference to address the issue. Accordingly, WARC-79 adopted a resolution calling for a world conference to be held no later than 1984 "to guarantee in practice for all countries equitable access to the geostationary satellite orbit and the frequency bands allocated to space services."

The debate at WARC-79 leading to the adoption of the resolution was contentious. Even though the regulatory procedures governing access to space services were retained basically unchanged, they were the subject of criticism by the developing countries. The present regulations were viewed by the developing countries as being based on the principle of "first come first served," and they feared that late comers might be denied access as the bands and orbit positions become more intensively used. On the other hand, the developed countries viewed the existing provisions as providing an orderly, flexible mechanism which naturally permits the introduction of technological advances-thereby insuring that the needs of all nations are met. Various proposals were introduced by developing nations proposing that a future WARC be convened to plan certain space services and bands.

In the ensuing debate, there was no disagreement as to the

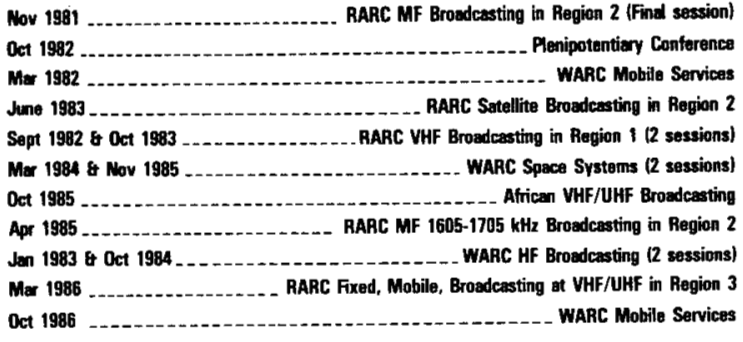

Fig. 1. A tentative schedule for future ITU conferences.

holding of a future conference. Instead, discussion centered on whether the conference should have a limited mandate restricted to producing a detailed plan along the lines adopted at the 1977 Broadcasting Satellite WARC, or whether the conference should have a broad mandate to consider all options and possibilities for guaranteeing equitable access. Particular concern was expressed that the 4/6-GHz Fixed-Satellite service was becoming congested, and that only by planning could the principle of equitable access to the spectrum-orbit resource be realized-as set forth in the ITU Convention. The WARC-77 approach specifies in great detail particular orbit positions, frequencies, and antenna beams to provide services to individual countries [7]. The developed countries, including those that supported BSS planning at the 1977 WARC, contended that detailed planning of the Fixed-Satellite service was neither feasible nor desirable, given the diverse nature of the service and that a detailed plan would freeze technological opportunities to expand the effective capacity of the orbit-spectrum resource.

In the end, agreement was reached on a two-stage conference that is to consider a range of possibilities to guarantee access to the geostationary-satellite orbit and space services utilizing it. The first session of the conference is to:

1) decide which space services and frequency bands should be planned (and establish planning principles, technical parameters, and criteria for any services and bands which may be so identified);

2) establish guidelines for regulatory procedures for services and bands that are not planned;

3) consider other possible approaches that meet the objective of ensuring guaranteed access to the orbit/spectrum. The second session of the conference is to implement the decisions taken at the first session.

\section{CONCLUSIONS}

Subsequent to WARC-79, the ITU Administrative Council at it's 1980 session considered the program of future conferences submitted to it by the conference. At that time, the council adopted a tentative schedule (see Fig. 1).

It can be seen that an extremely intensive schedule of activity has been proposed over a very compressed period of time. While WARC-79 unquestionably succeeded in identifying a full range of future conferences in accordance with it's mandate, one may reasonably ask whether the Council has spaced the conferences to allow Administrations and the permanent organs of the Union adequate time for preparation. The scheduling plans will need to be revised once the impact of the future conference workload becomes more evident as prepara- 
tory actions are initiated by Administrations and the ITU. The Plenipotentiary Conference of 1982 will reexamine this schedule and make further recommendations for implementation by the Administrative Council.

In addition to simply coping with the intensive workload outlined above, it is clear that finding solutions to questions of spectrum access posed by the broadcasting and space conferences discussed above will be a formidable challenge. One can readily agree to the general principle that all Administrations are entitled to equitable access to the radio spectrum, the problem nevertheless remains to define what is equitable as well as a practical means of achieving it, considering the diverse and disparate needs of Administrations.

\section{Note Added in Proof}

The Administrative Council, at its 1981 session, reviewed the entire schedule of future conferences and took the following action:

The RARC MF Broadcasting in Region 2 (final session), the RARC Satellite Broadcasting in Region 2, and the Plenipotentiary Conference were left as previously scheduled with very minor changes in dates.

All other conferences were rescheduled for dates which range between one and two years later than previously planned. The new dates are as follows:

Feb $83 \ldots \ldots \ldots \ldots$ WARC Mobile Services

Aug 82 and Oct $84 \ldots \ldots \ldots \ldots \ldots \ldots$
RARC VHF Broadcasting in Region 1 (2 sessions) July 85 and Sept 87 . . . WARC Space Systems (2 sessions) Jan $87 . \ldots \ldots$. . . . African VHF/UHF Broadcasting Sept $86 \ldots \ldots \ldots \ldots \ldots \ldots \ldots \ldots \ldots$ . . . RARC MF 1605-1705-kHz Broadcasting in Region 2 Jan 84 and Jan 86 . . WARC HF Broadcasting ( 2 sessions)

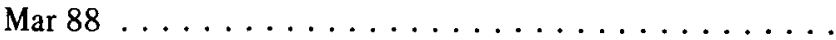
RARC Fixed, Mobile, Broadcasting at VHF/UHF in Region 3 Sept $88 \ldots \ldots \ldots \ldots \ldots$ WARC Mobile Services

\section{REFERENCES}

[1] ITU, "Agenda of the Conference," ITU/WARC-79 Doc. 1, Geneva, Switzerland, Sept. 29, 1978.

[2] ITÜ, "Final Protocol, Additional Protocols; Resolutions, Recommendations and Opinions," International Telecommunication Convention, Malaga-Torremolinos, Spain, 1973.

[3] ITU, "Recommendation 12 Relating to the Convening of Future Administrative Radio Conferences to Deal with Specific Services," in Final Acts of the World Administrative Radio Conference, Geneva, 1979. Geneva, Switzerland, 1979.

[4] ITU, "WARC for the Mobile Service," ITU/WARC-79 Doc. 6, Geneva, Switzerland.

[5] ITU, "Final Protocol No. 36," in Final Acts of the World Administrative Radio Conference. Geneva, Switzerland, 1979.

[6] ITU, "Procedure for the Bands Allocated Exclusively to the Broadcasting Service between $5950 \mathrm{kHz}$ and $26100 \mathrm{kHz}$," in Final Acts of the World Administrative Radio Conference. Geneva, Switzerland, 1979 , p. 226.

[7] ITU, Final Acts of the World Broadcasting-Satellite Administrative Radio Conference, Geneva, 1977. Geneva, Switzerland, 1977. 